\title{
A light in the dark sector with a Stueckelberg extension of the Standard Model
}

\author{
A.L. dos Santos and D. Hadjimichef \\ Instituto de Física, Universidade Federal do Rio Grande do Sul \\ Caixa Postal 15051, cep 91501-970, Porto Alegre, RS, Brazil
}

\begin{abstract}
In this work we present a double extension to the Standard Model (SM). One of the extensions is introduced, via minimal coupling, generating a massive $Z^{\prime}$ boson. This enlarged SM is coupled to a dark matter sector through the Stueckelberg mechanism by a $A^{\prime}$ boson. However, the $A^{\prime}$ boson does not interact directly with the SM fermions. In our study, we found that the $A^{\prime}$ is a massless photon-like particle in dark sector. We obtain a new coupling between the usual photon and the dark fermions. Constraints are made on its values.
\end{abstract}

Keywords: Stueckelberg, boson, dark sector

PACS: $12.60 .-\mathrm{i}, 95.35 .+\mathrm{d}$

\section{THE MODEL}

The model is a double extension of the Standard Model (SM). One of them is via minimal coupling and the second one is via Stueckelberg mechanism, where we introduce a pseudo-scalar field that decouple from the real fields and it don't interfere in the results. The model lagrangian density that represents this gauge group is written in a general form as a sum of

$$
\mathscr{L}=\mathscr{L}_{\mathrm{eSM}}+\mathscr{L}_{\mathrm{St}}
$$

where the enlarged Standard Model (eSM) part is defined by

$$
\begin{aligned}
\mathscr{L}_{\mathrm{eSM}}= & -\frac{1}{4} W_{\mu v}^{a} W^{a \mu v}-\frac{1}{4} B_{\mu v} B^{\mu v}-\frac{1}{4} C_{\mu \nu} C^{\mu v} \\
& -D_{\mu} \Phi^{\dagger} D^{\mu} \Phi-V\left(\Phi^{\dagger} \Phi\right)+i \bar{\psi} \gamma^{\mu} D_{\mu} \psi
\end{aligned}
$$

which represents a modified SM, obtained by introducing a new $U(1)$ boson field $C_{\mu}$ and the field tensor $C_{\mu \nu}=\partial_{\mu} C_{v}-\partial_{\nu} C_{\mu}$. This field is coupled to the other SM bosons in the covariant derivative [1]

$$
D_{\mu}=\partial_{\mu}+i g_{2} \frac{\tau^{a}}{2} W_{\mu}^{a}+i g_{Y} \frac{Y}{2} B_{\mu}+i g_{c} \frac{1}{2} C_{\mu}
$$

where $\Phi$ is the usual Higgs field. The Stueckelberg dark sector lagrangian is written as [2]

$$
\mathscr{L}_{\mathrm{St}}=-\frac{1}{4} X_{\mu \nu} X^{\mu \nu}-\frac{1}{2}\left(\partial_{\mu} \sigma+M_{1} C_{\mu}+M_{2} X_{\mu}\right)^{2}+\mathscr{L}_{\chi}
$$


where the new $U(1)$ vector boson in the dark sector is $X_{\mu}$ with its field tensor $X_{\mu \nu}=$ $\partial_{\mu} X_{v}-\partial_{\nu} X_{\mu}$. The second term in (4) is the Stueckelberg coupling term between the two boson fields $C_{\mu}$ and $X_{\mu}$ via an axial pseudo-scalar $\sigma$ field. The $\sigma$ field is unphysical and decouples from all fields after gauge fixing. The last term $\mathscr{L}_{\chi}$ is a general fermion term of the dark sector. This type of model was first proposed by [3], but with just one dark field, and was applied as well in [4]. Using this full Lagrangian (1) and taking into account just the terms that contribute to the bosons masses, we obtain

$$
\mathscr{L}^{\prime}=\ldots-\frac{1}{2} V^{\mu T} M^{2} V_{\mu},
$$

where $V^{\mu T}=\left(X^{\mu}, C^{\mu}, B^{\mu}, W^{3 \mu}\right)$ and the mass matrix is

$$
M^{2}=\left(\begin{array}{cccc}
M_{2}^{2} & M_{1} M_{2} & 0 & 0 \\
M_{1} M_{2} & M_{1}^{2}+\frac{g_{c}^{2} v^{2}}{4} & \frac{1}{4} g_{c} g_{Y} v^{2} & -\frac{1}{4} g_{2} g_{c} v^{2} \\
0 & \frac{1}{4} g_{c} g_{Y} v^{2} & \frac{g_{Y}^{2} v^{2}}{4} & -\frac{1}{4} g_{2} g_{Y} v^{2} \\
0 & -\frac{1}{4} g_{2} g_{c} v^{2} & -\frac{1}{4} g_{2} g_{Y} v^{2} & \frac{g_{2}^{2} v^{2}}{4}
\end{array}\right) .
$$

\section{TRANSFORMATION MATRIX}

Based on the matrix (6) we obtain an orthogonal matrix $O$, such that $O^{T} M^{2} O=M_{\text {diag }}^{2}$ and $O^{T} O=I$. This matrix is of the form

$$
O=\left(\begin{array}{cccc}
c_{\eta} s_{\phi} & c_{\phi} c_{\psi}+s_{\eta} s_{\phi} s_{\psi} s_{\theta} & c_{\phi} s_{\psi}-c_{\psi} s_{\eta} s_{\phi} s_{\theta} & -c_{\theta} s_{\eta} s_{\phi} \\
-c_{\eta} c_{\phi} & c_{\psi} s_{\phi}-c_{\phi} s_{\eta} s_{\psi} s_{\theta} & s_{\phi} s_{\psi}+c_{\phi} c_{\psi} s_{\eta} s_{\theta} & c_{\phi} c_{\theta} s_{\eta} \\
s_{\eta} & -c_{\eta} s_{\psi} s_{\theta} & c_{\eta} c_{\psi} s_{\theta} & c_{\eta} c_{\theta} \\
0 & c_{\theta} s_{\psi} & -c_{\psi} c_{\theta} & s_{\theta}
\end{array}\right),
$$

where $c_{\theta}=\cos \theta, s_{\theta}=\sin \theta$ and the angles are $\delta=\tan \phi=\frac{M_{1}}{M_{2}}, \tan \eta=\frac{g_{C}}{g_{y}} \cos \phi$, $\tan \theta=\frac{g_{Y}}{g_{2}} \sec \eta$ and $\tan \psi=\frac{M_{W}^{2} \sin \eta \tan \phi \sin \theta}{M_{W}^{2}-M_{Z^{\prime}}^{2} \cos ^{2} \theta}$.

After diagonalizing the matrix (6) we obtain 4 mass eigenvalues. They are

$$
\begin{aligned}
M_{Z^{\prime}}^{2}, M_{Z}^{2} & =\frac{1}{8}\left[v^{2}\left(g_{2}^{2}+g_{c}^{2}+g_{y}^{2}\right)+4 M_{1}^{2}+4 M_{2}^{2} \pm \Delta\right] \\
M_{\gamma}^{2} & =0 ; \quad M_{\gamma^{\prime}}^{2}=0 .
\end{aligned}
$$

where

$$
\Delta=\sqrt{8 M_{1}^{2}\left(4 M_{2}^{2}-v^{2}\left[g_{2}^{2}-g_{c}^{2}+g_{y}^{2}\right]\right)+\left(v^{2}\left[g_{2}^{2}+g_{c}^{2}+g_{y}^{2}\right]-4 M_{2}^{2}\right)^{2}+16 M_{1}^{4}} .
$$

We identify the masses with the $Z$ and $Z^{\prime}$ bosons masses. One of the zero eigenvalues can be associated, as usual, to the photon and the other one to a new massless boson, which we shall call a dark photon $\gamma^{\prime}$. 


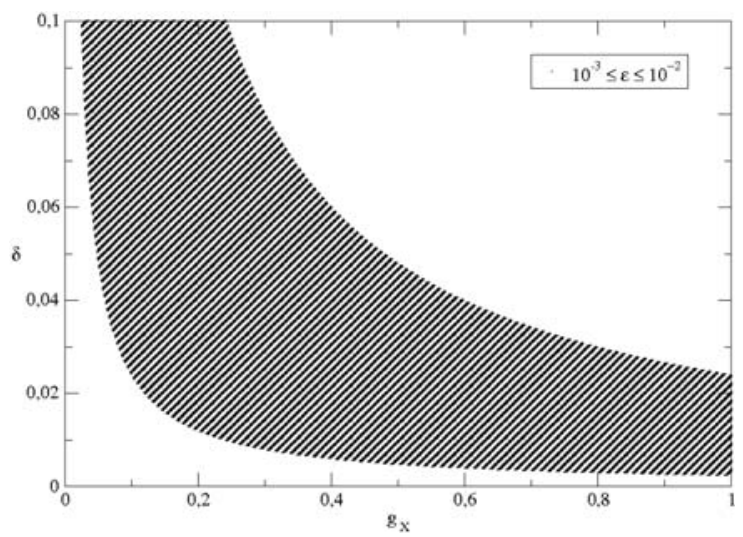

FIGURE 1. Region with the possible values for the coupling $g_{X}$ with respect to parameter $\delta$

Furthermore, the matrix $O$ mixes the boson fields, such that $U^{\mu}=O V^{\mu}$, where $V^{T \mu}=\left(X^{\mu}, C^{\mu}, B^{\mu}, W^{3 \mu}\right)$ and $U^{T \mu}=\left(A^{\prime \mu}, Z^{\prime \mu}, Z^{\mu}, A^{\mu}\right)$. By this mixing we redefine the coupling strength in which dark fermions couple to the standard model photon This can be written as

$$
e_{\chi}=\varepsilon e,
$$

where

$$
\varepsilon=\frac{g_{c} g_{X} \delta}{g_{c}^{2}+g_{Y}^{2}\left(1+\delta^{2}\right)}
$$

and $e$ is the eletric charge. In reference [5] we can see the possible values for $g_{c}$ and we assume that $e_{\chi}$ is a fraction of the order $\approx 10^{-3}$ of the eletric charge in the same way as obtained in reference [4]. In figure 1, we present the values of $g_{X}$ and $\delta$, that generate values of $1.0 \times 10^{-3} \leq \varepsilon \leq 1.0 \times 10^{-2}$. It is important to note that the result here agree with the same paramenters values in [4].

\section{ACKNOWLEDGMENTS}

This work was supported by CNPq.

\section{REFERENCES}

1. P. Langacker Rev. of Mod. Phys. 81 (2009) 3.

2. E. C. G. Stueckelber, Helv. Phys. Acta 11 (1938) 225; H. Ruegg and M. Ruiz-Altaba, Int. Jour. of Mod. Phys. A 19 (2004) 3265.

3. B. Körs and P. Nath, Phys. Lett. B 586 (2004) 366; and JHEP 07 (2005) 069.

4. K. Cheung Tzu-C. Yuan, JHEP 03 (2007) 120; Y. Zhang, S. Wang and Q. Wang, JHEP 03 (2008) 047; D. Feldman, Z. Liu, P. Nath and G. Peim, Phis. Rev. D 81 (2010) 095017.

5. A.L. dos Santos, D. Hadjimichef, arXiv:1207.6662v1. 
Copyright of AIP Conference Proceedings is the property of American Institute of Physics and its content may not be copied or emailed to multiple sites or posted to a listserv without the copyright holder's express written permission. However, users may print, download, or email articles for individual use. 